2012s-22

\title{
Déconcentration, délégation et dévolution : avantages, inconvénients et mise en place
}

\author{
Stéphanie Boulenger, Isabelle Gauthier, François Vaillancourt
}

Série Scientifique
Scientific Series

Montréal

Août 2012

(C) 2012 Stéphanie Boulenger, Isabelle Gauthier, François Vaillancourt. Tous droits réservés. All rights reserved. Reproduction partielle permise avec citation du document source, incluant la notice $\odot$.

Short sections may be quoted without explicit permission, if full credit, including (C) notice, is given to the source.
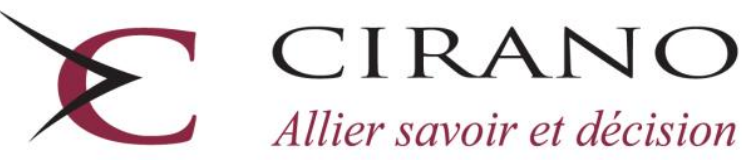

Allier savoir et décision

Centre interuniversitaire de recherche en analyse des organisations 


\section{CIRANO}

Le CIRANO est un organisme sans but lucratif constitué en vertu de la Loi des compagnies du Québec. Le financement de son infrastructure et de ses activités de recherche provient des cotisations de ses organisations-membres, d'une subvention d'infrastructure du Ministère du Développement économique et régional et de la Recherche, de même que des subventions et mandats obtenus par ses équipes de recherche.

CIRANO is a private non-profit organization incorporated under the Québec Companies Act. Its infrastructure and research activities are funded through fees paid by member organizations, an infrastructure grant from the Ministère du Développement économique et régional et de la Recherche, and grants and research mandates obtained by its research teams.

\section{Les partenaires du CIRANO}

\section{Partenaire majeur}

Ministère du Développement économique, de l'Innovation et de l'Exportation

\section{Partenaires corporatifs}

Autorité des marchés financiers

Banque de développement du Canada

Banque du Canada

Banque Laurentienne du Canada

Banque Nationale du Canada

Banque Royale du Canada

Banque Scotia

Bell Canada

BMO Groupe financier

Caisse de dépôt et placement du Québec

Fédération des caisses Desjardins du Québec

Financière Sun Life, Québec

Gaz Métro

Hydro-Québec

Industrie Canada

Investissements PSP

Ministère des Finances du Québec

Power Corporation du Canada

Rio Tinto Alcan

State Street Global Advisors

Transat A.T.

Ville de Montréal

\section{Partenaires universitaires}

École Polytechnique de Montréal

HEC Montréal

McGill University

Université Concordia

Université de Montréal

Université de Sherbrooke

Université du Québec

Université du Québec à Montréal

Université Laval

Le CIRANO collabore avec de nombreux centres et chaires de recherche universitaires dont on peut consulter la liste sur son site web.

Les cahiers de la série scientifique (CS) visent à rendre accessibles des résultats de recherche effectuée au CIRANO afin de susciter échanges et commentaires. Ces cahiers sont écrits dans le style des publications scientifiques. Les idées et les opinions émises sont sous l'unique responsabilité des auteurs et ne représentent pas nécessairement les positions du CIRANO ou de ses partenaires.

This paper presents research carried out at CIRANO and aims at encouraging discussion and comment. The observations and viewpoints expressed are the sole responsibility of the authors. They do not necessarily represent positions of CIRANO or its partners. 


\title{
Déconcentration, délégation et dévolution : avantages, inconvénients et mise en place*
}

\author{
Stéphanie Boulenger ${ }^{\dagger}$, Isabelle Gauthier ${ }^{*}$, François Vaillancourt ${ }^{\S}$
}

\section{Résumé / Abstract}

L'objectif de ce texte est de présenter les trois différentes formes de décentralisation (décentralisation administrative, politique et fiscale) et les trois différents types de décentralisation, soit la déconcentration, la délégation et la dévolution. On fait ressortir les forces et faiblesses des trois types et examine trois aspects spécifiques de la mise en place de la décentralisation. Ce texte se divise en trois sections, répondant aux trois questions suivantes: «Qu'est-ce que la décentralisation?», «Comment choisir un type de décentralisation? », «Comment gérer et mettre en place la décentralisation? »

Mots clés : déconcentration, délégation, dévolution, décentralisation.

The purpose of this paper is to present the various forms (administrative, political and fiscal) and the various types (deconcentration, delegation, devolution) of decentralization. We highlight the strength and weaknesses of these various forms of decentralisation, as well as examine the specific aspects of their implementation. We divide this text into three sections, based on the following questions : "What is decentralisation?", "How do we choose the type of decentralisation we want?" and "How do we manage decentralisation?"

Keywords: deconcentration, delegation, devolution, decentralisation.

\footnotetext{
* Texte dont la première version fut préparée pour l'Institut de la Banque Mondiale.

${ }^{\dagger}$ Directrice de recherche CIRANO.

\$ économiste Communauté Métropolitaine de Montréal (CMM).

${ }^{\S}$ Fellow CIRANO et professeur associé, sciences économiques, Université de Montréal, courriel : francois.vaillancourt@umontreal.ca
} 


\section{Introduction}

L'objectif de ce texte est de présenter les trois différents types de décentralisation, soit la déconcentration, la délégation et la dévolution, et de faire ressortir les forces et faiblesses de ces différents types. De plus, ce texte examine trois aspects spécifiques de la gestion et de la mise en place de la décentralisation et, en particulier, les trois dimensions de la dévolution (administrative, politique et fiscale). Ceci est pertinent, car la décentralisation est une mesure utilisée dans bon nombre de pays pour améliorer les prestations du secteur public. Ceci s'explique par le fait que lorsque la décentralisation est bien préparée et mise en œuvre judicieusement, elle amène une amélioration de la prestation de services suite à une meilleure prise en compte des besoins des résidents par les décideurs des entités décentralisées. Ce texte se divise en trois sections, répondant aux trois questions suivantes : "Qu'est-ce que la décentralisation?», "Comment choisir un type de décentralisation? » et « Comment mettre en place la décentralisation?».

\section{Qu'est-ce que la décentralisation?}

La décentralisation est un phénomène relié au transfert de pouvoir des organes de décision de l'état central œuvrant dans la capitale vers soit des organes de l'état central hors capitale, soit un autre niveau de gouvernement ou autorité, ou soit des gouvernements sous- nationaux (cantons, états, provinces...) ou locaux (sn/l); ceci est vrai aussi bien pour les états unitaires que pour les états fédérés. Les trois types de décentralisation sont: la déconcentration, la délégation et la dévolution. Notons qu'ils sont présentés à la fois par ordre alphabétique et par ordre croissant de réduction de pouvoir de l'état central. Notons aussi que nous adoptons une perspective dans laquelle les pouvoirs appartiennent tous à l'État central - où une assemblée constitutionnelle choisit la répartition de ces pouvoirs entre divers niveaux de gouvernements. Une autre perspective est que des entités se regroupent en un pays et choisissent quels pouvoirs transférer (concentrer) vers l'État central - comme, par exemple, lors de la création du Canada ou États-Unis. 


\section{Déconcentration}

La déconcentration, signifie que l'état central garde ses pouvoirs et responsabilités pour une fonction spécifique, mais fait exercer/exécuter cette fonction en dehors de la capitale par des antennes ou bureaux de l'administration situés en région. Un tel système peut être mis en place par la responsabilisation des gouverneurs ou préfets dont les bureaux se situent en dehors de la capitale, ou par la création de directions régionales ou préfectorales de ministères ou par les deux mécanismes, ce qui soulève alors des questions de coordination et de partage d'autorité. L'important est que les fonctionnaires et cadres situés en région puissent prendre des décisions sans en référer à la capitale et ne soient pas de simples courroies de transmission.

Le terme déconcentration peut être défini comme étant la répartition territoriale des tâches, permettant de régler le plus rapidement possible les problèmes dont s'occupe l'administration, grâce, notamment, à une compréhension plus directe des conditions locales par les organes déconcentrés disposant de la compétence de décision. Selon Delcamp (1995), la décentralisation territoriale, comme il l'appelle, signifie: "Un transfert d'un pouvoir de décision ou d'une responsabilité d'exécution à une entité administrative au sein de la même personne morale ».

Ainsi, les organes centraux restent compétents pour l'orientation générale et pour la coordination. Suivant ces principes, leurs compétences comprennent l'orientation générale, la coordination et le contrôle des organes déconcentrés. Cependant, ce sont les organes déconcentrés qui bénéficient de la présomption de compétence, dans la mesure où ils disposent de la compétence de décision générale sur les affaires de leurs circonscriptions, les organes centraux se limitant aux compétences qui leur sont attribuées par des dispositions spéciales qui se rapportent à un objet précis. Les principes de la déconcentration reçoivent, sous des formes diverses, une reconnaissance constitutionnelle dans quelques constitutions européennes (Delcamp, 1995). 


\section{Délégation}

La délégation se produit lorsque l'état central garde ses responsabilités et compétences, mais délègue la prestation et l'administration des services à un gouvernement sous-national (provinces, cantons,...) ou à l'autorité locale. Insistons ici qu'il s'agit d'un gouvernement autonome qui est le récipiendaire de la compétence délégué et non pas une entité déconcentrée de l'état central; il y a donc des politiciens différents de ceux gérant l'état central qui sont responsables auprès des citoyens de la gestion des services offrant la prestation déléguée. Cette délégation de compétences peut se faire non seulement par l'État, mais aussi par les gouvernements des régions; trop de délégation peut entrainer une complexité, peu propice à l'autonomie locale (Delcamp, 1995).

Bolderson et Mabbett (1999), qui utilisent le modèle de principal-agent pour fin d'analyse, indiquent que la délégation de compétences vers les gouvernements sn/l permet d'améliorer l'efficacité de l'administration des services. Ainsi, si les fonds nécessaires sont disponibles au niveau sous-national/local, en d'autres mots si le problème des responsabilités non financées ("unfunded mandates») est écarté, l'administration des services se fera adéquatement et permettra aux gouvernements $s n / l$ d'atteindre un certain degré d'autonomie dans la prestation des services délégués. L'utilisation du modèle principal-agent est intéressante car la principale difficulté que rencontre le principal dans ce modèle provient d'un manque d'information sur les capacités, coûts et activités de l'agent. Il cherche à pallier ce manque d'information en écrivant un contrat approprié assurant que l'agent exécute bien sa fonction.

Les compétences déléguées s'accompagnent malheureusement quelquefois du phénomène de dédoublement fonctionnel, c'est-à-dire, que puisque les fonctions ne sont pas bien définies entre les niveaux de gouvernement, ceci entraine une certaine confusion et inefficacité aussi bien au sein des gouvernements que chez les utilisateurs. (Delcamp, 1995). 


\section{Dévolution}

La dévolution, soit le niveau de décentralisation associé à la plus importante réduction des pouvoirs de l'état central, s'accompagne du transfert des responsabilités et compétences de celui-ci aux gouvernements sn/l. La littérature française utilise fréquemment le terme décentralisation pour dénoter la dévolution, ce qui est quelquefois une source de confusion pour ceux s'initiant à cette question. Ce transfert signifie que l'état central perd tout droit de regard sur la quantité et qualité des services offerts et sur les modes de prestation. C'est le cas des responsabilités attribuées aux provinces canadiennes et cantons suisses. Ainsi, il n'y a pas de ministère national de l'éducation dans ces pays, car c'est une responsabilité des sn/l. On peut cependant avoir dévolution avec normes nationales, ce qui oblige à une certaine qualité/quantité de prestations. On fait alors face à un mélange délégation/dévolution avec des compositions variantes selon les pays et responsabilités. C'est le cas des États-Unis où le gouvernement fédéral est plus présent dans les champs de compétence étatiques par ses programmes de transfert aux nombreuses conditions de financement.

Pour Rosenbaum (1998), le concept de dévolution est un peu moins clairement défini, en partie parce qu'il est récent. L'une des choses qui aurait contribué au malentendu serait la confusion entre la dévolution, la délégation et la déconcentration. Rosenbaum (1998) définit la dévolution comme suit: "la décentralisation véritable comprend la délégation des responsabilités et des ressources à des autorités infra-nationales relativement indépendantes et autonomes qui sont responsables non pas devant le pouvoir central, mais devant les citoyens de la région ou de la communauté. » Selon lui, un élément important du mouvement de dévolution a été le renforcement, voire la mise en place, de gouvernements intermédiaires, soit les provinces, régions, états,.. . Ce mouvement permettrait d'encourager la participation des citoyens et groupes, et au développement des organisations communautaires dans les zones aussi bien rurales qu'urbaines. 


\section{Essai de comparaison des 3D}

Le tableau 1 présente chacun des types de décentralisation sous l'angle de trois aspects: les responsables politiques, les responsables de l'exécution et les responsables du financement. Ce tableau permet d'avoir une vue d'ensemble de la situation et donc de faire ressortir les différences et similarités entre les trois types de décentralisation. L'examen du Tableau 1 nous indique que c'est la délégation qui est la forme la plus complexe de décentralisation.

Tableau 1 : Trois aspects des trois types de décentralisation

\begin{tabular}{|c|c|c|c|}
\hline Types & $\begin{array}{c}\text { Responsables } \\
\text { politique }\end{array}$ & $\begin{array}{c}\text { Responsables de } \\
\text { l'exécution }\end{array}$ & $\begin{array}{l}\text { Responsable du } \\
\text { financement }\end{array}$ \\
\hline Déconcentration & Élus nationaux & $\begin{array}{c}\text { Employés de l'état } \\
\text { central }\end{array}$ & Budget national \\
\hline Délégation & $\begin{array}{c}\text { Élus nationaux } \\
\text { et } \\
\text { Élus sn/l }\end{array}$ & $\begin{array}{c}\text { Employés du } \\
\text { gouvernement sn/l } \\
\text { Supervisés par } \\
\text { employés de l'état } \\
\text { central } \\
\end{array}$ & $\begin{array}{c}\text { Budget sn } / \mathrm{l} \text {, avec ou } \\
\text { sans } \\
\text { Paiements } \\
\text { contractuels de l'état } \\
\text { central, venant du } \\
\text { Budget national }\end{array}$ \\
\hline Dévolution & Élus sn/l & $\begin{array}{c}\text { Employé du } \\
\text { gouvernement sn/l } \\
\text { (incluant } \\
\text { Corps d'employés } \\
\text { nationaux) }\end{array}$ & $\begin{array}{c}\text { Budget sn/l } \\
\text { Taxes ou } \\
\text { Transferts de l'état } \\
\text { central venant du } \\
\text { Budget national }\end{array}$ \\
\hline
\end{tabular}

Source : Auteurs

Revenons sur les trois colonnes du tableau 1 pour approfondir ces concepts dans le cas de la décentralisation avancée - soit la dévolution.

\section{Dévolution/décentralisation politique}

La décentralisation politique repose sur l'élection, par les résidents d'entités territoriales sous-nationales, des décideurs sn/l : conseillers, maires, gouverneurs etc. Ceci implique souvent de remplacer des décideurs nommés par l'État central par des décideurs élus. Être un décideur implique pouvoir faire des choix significatifs et, en particulier, des choix de dépenses et de recettes. Ceci peut exiger des amendements constitutionnels ou encore le renforcement des lois ou des 
réformes électorales au niveau sn/l. Par ailleurs, ceci peut exiger la création d'assemblées législatives $s n / l$ ou la mise en place de réformes constitutionnelles qui renforcent l'autonomie politique des gouvernements sn/l (Falleti, 2005).

Les défenseurs de la décentralisation politique avancent que les décisions prises seront mieux informées et répondront mieux aux intérêts divers de la société que celles prises par les autorités politiques centrales. La décentralisation politique permet aux citoyens de mieux connaître leurs représentants politiques et permet aux élus de mieux connaître les besoins et les désirs de leurs électeurs. (Banque Mondiale, 2011). Pour renforcer le lien de redevabilité, les élections sont préférablement directes et non pas indirectes.

\section{Dévolution/Décentralisation administrative}

La décentralisation administrative veut donner les moyens, autres que financiers, aux gouvernements $s n / l$, pour assurer la fourniture de services publics, tels l'éducation et la santé. Elle donne le pouvoir aux gouvernements $s n / l$ d'acquérir les divers intrants nécessaires à cette fourniture de services. En particulier, les gouvernements $s n / l$ peuvent embaucher et licencier du personnel ainsi qu'en fixer la rémunération, sans besoin de se référer au niveau supérieur de gouvernement. (Shah \& Thompson, 2004).

\section{Dévolution/ Décentralisation fiscale}

La décentralisation fiscale se réfère à l'ensemble des politiques créées afin d'accroître les revenus et l'autonomie fiscale des gouvernements $s n / l$. (Shah \& Thompson, 2004). Ces politiques sont nombreuses: a) l'autofinancement ou le recouvrement des coûts par l'imposition de frais aux usagers; b) le co-financement ou la co-production où les usagers participent en fournissant des services et infrastructures via des contributions monétaires ou en nature (par le travail); c) la mise en place d'impôts fonciers, de taxes de vente ou de charges indirectes; d) les transferts inter-gouvernementaux où les revenus en impôts collectés par le gouvernement central sont transférés aux gouvernements $s n / l$; e) l'autorisation 
d'emprunter et la mobilisation de ressources locales ou nationales pour des garanties de prêt.

Notons cependant que dans beaucoup de pays en voie de développement, les gouvernements les unités administratives locales possèdent l'autorité légale pour taxer ou prélever des impôts, mais l'assiette fiscale étant trop petite et la dépendance vis-à-vis les subventions du gouvernement central tellement implantée dans les habitudes politiques que les gouvernements locaux n'exercent souvent pas cette autorité. (Banque Mondiale, 2011)

Pour une décentralisation fiscale efficace, il faut donc garantir une source adéquate et autonome des revenus ainsi que l'autonomie des dépenses et le privilège d'emprunt, ce dernier étant un peu plus circonscrit (Shah \& Thompson, 2004). Si des revenus sont transférés du gouvernement central vers le gouvernement sn/l afin de couvrir les coûts de la fourniture de services, la dévolution est financée. Si les gouvernements $s n / l$ doivent couvrir leurs coûts avec leurs propres revenus, on dit que la dévolution n'est pas financée (Falleti, 2005).

Une fois que les types de décentralisation sont bien définis, il faut trouver celle qui permet le mieux d'améliorer la qualité des services publics / d'en accroître la quantité offerte. Ainsi, la deuxième question est : «Comment choisir le bon type de décentralisation? ».

\section{Comment choisir un type de décentralisation?}

Le choix de l'un ou l'autre type de décentralisation relève de la compétence de chaque état. Il n'y a pas de réponse standard; chaque choix reflète un ensemble de facteurs et en particulier leur dimension spatiale soit la géographie (taille, topographie), l'histoire, la démographie (composition ethnique/ linguistique/ religieuse) l'économie et le cadre légal et la répartition spatiale de ces composantes. (Bird \& Vaillancourt, 1998; Vaillancourt 2011) 
Une façon d'effectuer un choix éclairé est d'examiner les avantages et les inconvénients des trois types de décentralisation, et de choisir celui qui apporte le plus d'avantages nets des inconvénients. Le tableau 2 présente les avantages et inconvénients de la décentralisation mis de l'avant par Rosenbaum (1998) et leur appariement par nos soins avec les trois types de décentralisation. Nous évaluons les avantages et inconvénients comme n'existant pas (nil), ou comme étant faible, moyen ou élevé. Cette échelle s'interprète en termes de chaque avantage ou inconvénient étudié - chaque lecteur doit décider lui-même quel poids attribuer à chaque élément.

\section{Tableau 2 : Avantages et Inconvénients des trois types de décentralisation}

\begin{tabular}{|c|c|c|c|}
\hline & Déconcentration & Délégation & Dévolution \\
\hline \multicolumn{4}{|l|}{ AVANTAGES } \\
\hline $\begin{array}{l}\text { A.1. Fragmenter et disperser le pouvoir } \\
\text { politique }\end{array}$ & Nil & Faible & Élevé \\
\hline $\begin{array}{l}\text { A.2. Créer un espace civique } \\
\text { supplémentaire }\end{array}$ & Nil & Faible & Élevé \\
\hline $\begin{array}{l}\text { A.3. Émergence de groupes politiques } \\
\text { d'opposition }\end{array}$ & Nil & Faible & Moyen \\
\hline $\begin{array}{l}\text { A.4. Création de terrains } \\
\text { d'apprentissage pour le } \\
\text { développement de compétences et de } \\
\text { pratiques démocratiques }\end{array}$ & Nil & Moyen & Moyen \\
\hline $\begin{array}{l}\text { A.5. Permet plus d'options pour les } \\
\text { citoyens pour des services particuliers }\end{array}$ & Nil & Faible & Élevé \\
\hline $\begin{array}{l}\text { A.6. Adaptation à la diversité des } \\
\text { attentes populaires }\end{array}$ & Faible & Moyen & Élevé \\
\hline $\begin{array}{l}\text { A.7. Efficacité politique pour } \\
\text { l'ensemble des citoyens des localités }\end{array}$ & Faible & Faible & Moyen \\
\hline $\begin{array}{l}\text { A.8. Accroissement des } \\
\text { possibilités d'initiative de l'économie } \\
\text { locale }\end{array}$ & Faible & Moyen & Élevé \\
\hline \multicolumn{4}{|l|}{ INCONVÉNIENTS } \\
\hline $\begin{array}{l}\text { I.9. Irresponsabilité fiscale car } \\
\text { financement par transfert et non par } \\
\text { des impôts locaux }\end{array}$ & Élevé & Moyen & Faible/Nil* \\
\hline I.10. Capture par l'élite locale & Faible & Moyen & Élevé \\
\hline $\begin{array}{l}\text { I.11. Risque de perspectives étroites } \\
\text { des instances locales en termes de } \\
\text { politiques publiques que l'état central }\end{array}$ & Nil & Moyen & Élevé \\
\hline
\end{tabular}




\begin{tabular}{|l|c|c|c|}
\hline & Déconcentration & Délégation & Dévolution \\
\hline $\begin{array}{l}\text { I.12. Adéquation des responsabilités } \\
\text { des ressources }\end{array}$ & Nil & Moyen/Élevé* & Moyen/Élevé* \\
\hline
\end{tabular}

Note : * Tout dépendant du mode de financement (transferts ou taxation autonome)

Source : Rosenbaum (1998), p. 509-514 et auteurs.

Selon Rosenbaum, le principal avantage de la décentralisation est qu'elle constitue un moyen de fragmenter et disperser le pouvoir politique (item A.1 tableau 2). Selon lui, il est important d'établir et de maintenir un système d'équilibre de contre-pouvoirs. La dévolution représente le meilleur moyen pour réaliser un tel équilibre. En dispersant le pouvoir, il y a création d'indépendance et d'autonomie, permettant de réduire une concentration excessive du pouvoir et de l'autorité au niveau central. L'à, - propos d'un tel objectif dépend en partie du degré d'appartenance nationale que ressentent les divers groupes au sein d'un pays/état donné. Il y a un degré optimal de décentralisation qui tient compte, sans les attiser, des différences intergroupes bien qu'il soit difficile à établir (Bird et Vaillancourt, 2009).

On note au point A.2 du tableau 2, la création d'un espace civique supplémentaire, c'est-à-dire que l'autonomie attribuée au niveau d'autorité sn/l contribue à renforcer la responsabilité des gouvernements à l'égard de la société civile. Dans le même ordre d'idée, l'avantage présenté au point A.3 du tableau 2, se produit lorsqu'il y a mécontentement de la société et mobilisation de celle-ci par la création de groupes politiques d'opposition avec les ressources nécessaires pour leur organisation efficace. Ces deux avantages émergent comme résultat de la dévolution. Le fait que les responsabilités soient transférées à l'autorité $s n / l$ permet d'atteindre cet objectif. Par contre, la déconcentration ne permet pas ceci et la délégation est peu susceptible d'arriver à un tel résultat, car les liens de dépendance face à l'état central sont usuellement trop grands.

$\mathrm{Au}$ point A.4 du tableau 2, on traite de La création de nombreux terrains d'apprentissage pour le développement de compétences et de pratiques démocratiques. Rosenbaum (1998) prétend que dans les pays en transition, où il n'y a pas de tradition de participation démocratique, ceci est particulièrement important, car cela fournit une arène où ceux qui n'ont pas fait partie de l'ancienne 
élite peuvent commencer à développer leurs propres compétences et expériences politiques. À la marge, la déconcentration ne permet pas d'améliorer le système démocratique d'un pays. Par contre, la délégation et la dévolution permettent de profiter de terrains d'apprentissage plus ou moins encadrés.

Les avantages de la décentralisation recensés aux points A.5 et A.6, du tableau 2, se complètent tout particulièrement. En transférant des responsabilités aux localités ou aux instances sous-nationales, l'état central permet aux citoyens d'obtenir une plus grande gamme de choix de services selon les localités. La décentralisation permet ainsi, de combiner une certaine mesure d'uniformité avec la possibilité de faire des adaptations locales et d'être plus en résonance avec les besoins et les intérêts de la population (Rosenbaum, 1998). Ainsi, plus le type de décentralisation est poussé, plus cet avantage est présent.

Le septième avantage de la décentralisation, noté en A.7 au tableau 2, est qu'elle donne un sentiment plus grand d'efficacité politique à l'ensemble des citoyens. D'une manière générale, ceux-ci tendent à réagir plus positivement à un gouvernement plus proche d'eux, même si les politiques poursuivies par le gouvernement ne sont pas les plus favorables aux individus concernés. En d'autres mots, plus l'autorité est près du citoyen plus il sera en confiance, ce qui facilitera la perception fiscale.

Enfin, au point A.8 du tableau 2, on avance que la décentralisation offre davantage de possibilités à l'initiative économique locale, car les communautés éloignées de la capitale pourront alors mettre de l'avant leurs avantages et besoins particuliers plutôt que ceux de la nation dans son ensemble.

Rosenbaum identifie quatre inconvénients de la décentralisation qui se retrouvent également au tableau 2 .

Le premier inconvénient (soit I.9), souligné par l'auteur, est le risque d'irresponsabilité fiscale. Les dangers sont particulièrement élevés lorsque les responsables locaux n'ont pas la responsabilité de collecter leurs propres recettes mais profitent de ressources qui leur sont cédées par un autre niveau de gouvernement sous forme de transferts. De plus, le problème des responsabilités non financées, c'est-à-dire que l'état central transfère une responsabilité à l'autorité 
sn/l sans la financer adéquatement, peut faire surface. Le type de décentralisation qui est le plus touché par ce risque est la délégation, puisqu'ici l'autorité $s n / l$ a la responsabilité d'administrer le service mais est plus ou moins financé par l'état central.

Le deuxième inconvénient, (I.10 au tableau 2), est que les instances locales peuvent être capturées par des élites locales bien organisées même si disposant qu'une base très étroite au sein de la population. En d'autres mots, la décentralisation risque d'entraîner un contrôle excessif de l'autorité locale par les élites locales. Ceci est particulièrement préoccupant au sein d'un état où la démocratie est implantée de façon inégale d'une région à l'autre.

Le troisième inconvénient suggéré par Rosenbaum (I.11 au tableau 2) est le risque que les sn/l est une perspective plus étroite en termes de politiques publiques que celle du gouvernement central. Ainsi, des efforts pour réaliser une politique d'intérêt général peuvent, délibérément ou non, être contrecarrés par les actions des autorités locales (Rosenbaum, 1998). Ceci peut réduire les droits de certains groupes défavorisés.

Enfin, (I.12 au tableau 2) la décentralisation peut servir de façade pour permettre au gouvernement central de se départir de la responsabilité de fournir des services importants. Ceci se produit si l'on transfère les responsabilités à des autorités locales sans leur fournir les ressources nécessaires. Ainsi, il y aura alors émergence du problème de l'adéquation des responsabilités et des ressources pour les financer.

Une fois un choix fait par un état, surgit la question de la gestion de la décentralisation. C'est là l'objet de la dernière section du texte.

\section{Comment gérer la nouvelle forme de décentralisation?}

Lorsque le type de décentralisation a été choisi, il faut déterminer quels sont les moyens ou les stratégies pour réussir une saine gestion de celle-ci. À cet égard, il nous semble utile de se pencher sur trois questions : soit l'organisation du territoire, la fonction publique et le financement. 


\subsection{Organisation du territoire}

Le découpage territorial d'un pays joue un grand rôle dans la mise en place de toute décentralisation. Selon Rémond (1999): «Partager ou fragmenter le tissu territorial ou les responsabilités locales pour mieux gouverner, au niveau central, l'ensemble du territoire: cette orientation a profondément marqué et marque encore le découpage territorial, le fonctionnement des institutions locales et le financement des collectivités territoriales françaises. » Bien avant le 19e siècle, l'État, qu'il s'agisse des responsables politiques ou des décideurs administratifs - que ce soit en République ou en régime monarchique, a toujours préféré, sinon la multitude de petites entités, du moins un découpage territorial qui fragmente le paysage institutionnel et politique afin d'éviter que ne se dressent face à lui des pouvoirs locaux trop puissants (Rémond, 1999). Bien que compréhensible, compte tenu des conditions historiques et idéologiques qui marquent la naissance d'un nouvel État, pour montrer l'indivisibilité et l'unité de l'État, cette option n'est plus recommandable dans un contexte de vraie décentralisation.

Rémond rajoute que plusieurs niveaux de collectivités locales engendrent des conséquences négatives qui nuisent à la pleine réalisation de la décentralisation quelque soit le type choisi par l'État : Selon lui,

- Un État qui dénombre quatre ou même cinq niveaux de gestion territoriale ne peut que favoriser le maintien et la prééminence de la pression étatique du centre. Un découpage territorial aussi complexe, même s'il s'accompagnait d'une répartition très rationnelle des compétences et des attributions entre chaque niveau, ne peut que faire le jeu de l'État central, seul à être uniformément présent partout et seul à avoir une vision générale de chacun des problèmes soulevés.

- Même si de nombreux niveaux de collectivités locales semblent être une richesse démocratique, le transfert de compétences à des communes, qui n'ont guère les capacités techniques, financières ou économiques, ne peut leur donner un sentiment d'autonomie. En effet, il n'y a que transfert de la tutelle de l'État vers une autre forme 
de tutelle. Le citoyen ne peut identifier clairement les responsabilités en matière de services publics de chaque niveau.

- L'existence de plusieurs niveaux de collectivités locales ne peut permettre d'imaginer des collectivités territoriales fortes: elles seront plutôt faibles. La décentralisation veut remédier aux méfaits d'une structure administrative et territoriale jugée trop pyramidale. Elle veut ainsi créer une architecture territoriale horizontale composée de collectivités autonomes dans leur sphère de compétences, sans tutelle d'une collectivité sur une autre, ni hiérarchie entre elles. Mais l'inégalité qui caractérise la situation de chacune de ces collectivités, l'une par rapport à l'autre, et l'ensemble de ces mêmes collectivités par rapport à l'État, ne peut que favoriser le maintien ou la réapparition, sous d'autres formes, de la tutelle et du pouvoir hiérarchique de l'État central. (Rémond, 1999)

Il est par contre important de noter que dans un contexte de décentralisation bien conçue, ces problèmes ne surgissent pas et que l'État n'est pas opposé aux collectivités territoriales - l'État étant à la fois national et territorial. De même, l'intérêt national et les intérêts locaux ne sont pas en opposition : les pouvoirs publics ont en charge l'intérêt des deux.

Selon Rémond (1999) il faut, dans le cas de la France, redéfinir les institutions en fonction des réalités de la décentralisation : «Procéder en sens inverse et garder des découpages anciens et tronqués, en espérant que les responsables politiques placés à leur tête pourraient _... régler les problèmes de politiques publiques posés, est voué à l'échec. »

Selon nous, les questions soulevées ci-haut, bien que posées dans le contexte français, sont d'applications générales. Lorsqu'il y a décentralisation, il faut examiner si la structure territoriale en place demeure appropriée, et en particulier, si elle n'est pas trop fragmentée compte tenu des nouvelles responsabilités de certaines entités et de la disponibilité du personnel nécessaire à la supervision et à l'exécution des tâches. 


\subsection{L'organisation de la fonction publique}

L'organisation de la fonction publique d'un État qui entreprend une réforme de décentralisation doit s'appuyer sur un certain nombre d'orientations et tenir compte des questions suivantes : «Qui travaillera au niveau sn/l? » et «Comment se fera la coordination des organes déconcentrés? » (Rosenbaum, 1998).

La première question cache un ensemble de questions subsidiaires, à savoir :

- Si l'on utilise la déconcentration, devra-t-on déplacer des fonctionnaires de l'état central en région ou en recruter sur place? Si on doit les déplacer, qui décidera les affectations? Est-ce que se seront les bureaux centraux de l'état qui ont intérêt à garder les meilleurs éléments pour eux ou consultera-t-on les décideurs locaux pour choisir conjointement les fonctionnaires déconcentrés;

- Si l'on utilise la délégation, qui recrutera les employés locaux et que se produira-t-il si les fonctions sont reprises plus tard par l'état central? Si cette délégation entraîne des pertes d'emplois au niveau central, ces employés ont-ils un accès prioritaire aux emplois créés par la délégation?

- Si l'on utilise la dévolution, les deux questions soulevées par l'utilisation de la délégation se posent.

Dans certains pays, certains fonctionnaires locaux sont des employés de l'état (secrétaire général, comptable municipal, etc.) dont le salaire émarge au budget de la municipalité mais dont la loyauté est avant tout à l'état central. Ceci implique que leurs salaires et avantages sociaux (retraite) ne sont pas fixés par leur employeur et surtout qu'ils sont susceptibles d'entrer en conflit avec les tenants politiques de la décentralisation.

De façon plus générale, la bureaucratie et la fonction publique ne doivent pas être jugées sur les intrants, mais bien sur les résultats. En d'autres termes, un système de gestion public sera jugé sur l'action concrète, sur le rapport coût efficacité de ses activités et sur la juste utilisation des ressources publiques. Le contrôle financier d'un tel système impose une comptabilité publique obligeant à rendre compte de l'utilisation des ressources publiques et documentant les décisions en matière d'allocation (Banque Mondiale, Bénin, 2002). Il faut un système 
de rémunération de la fonction publique qui a pour objectif d'assurer que la politique salariale soit cohérente avec la productivité et que les augmentations de salaire reflètent la performance. De même, le système de promotion reposera sur la performance, laquelle sera évaluée selon des objectifs établis annuellement avec la participation des fonctionnaires, ce qui impliquera une décentralisation de la gestion du personnel au niveau de chaque ministère et de chaque unité au sein de l'administration. De plus, pour avoir la possibilité de réaliser pleinement le processus de décentralisation, il faut que la gestion de la fonction publique soit à la mesure des nouvelles responsabilités qui lui sont délivrées.

La deuxième question se pose uniquement dans le cas de la déconcentration. Il s'agit de savoir si la coordination des actions déconcentrées se fera au niveau central, là où se faisait la coordination des activités concentrées auparavant, ou si elle se fera au niveau sn/l. Dans ce deuxième cas, il faut identifier un coordonnateur. On propose souvent le responsable territorial soit le gouverneur, préfet ou souspréfet. Mais un tel choix implique qu'un employé d'un ministère, usuellement l'Intérieur ou son équivalent, ou relevant du premier ministre/président soit le supérieur pour certaines décisions des employés d'autres ministères, ce qui cause souvent des problèmes. La réponse est qu'il n'y a pas de solution unique à cette question; chaque pays doit en trouver une adaptée à sa réalité.

\section{3. $\quad$ Financement et budgétisation de la décentralisation}

Ici, il faut distinguer les trois types de décentralisation et les questions budgétaires et de flux de financement effectivement versé.

\section{Budgétisation}

La question de l'inscription budgétaire des montants associés à la décentralisation se pose surtout pour la déconcentration car il s'agit d'inscrire au budget central des fonds qui seront déboursés par des organes déconcentrés de l'état central. La possibilité de ce faire dépend du libellé de la loi de gestion financière. Une question va être le degré de détail du budget de l'entité déconcentrée; reproduira-t-on le niveau de détail du budget central ou laissera-t-on au responsable budgétaire une certaine flexibilité? 
Dans le cas de la délégation et de la dévolution, il s'agit d'inscrire le montant à payer comme dépense de l'état sous forme de transfert aux sn/l. Ceci étant un type de déboursé, cette façon de faire rencontre moins de difficultés administratives.

\section{Flux financiers}

Dans le cas de la déconcentration, les ressources de l'état central sont redéployées au sein de son budget mais les règles budgétaires demeurent inchangées.

Dans le cas de la délégation, il s'agit d'un contrat de fourniture de services entre l'état central acheteur et le $s n / l$, fournisseur, dont l'une des composantes est le prix ou coût des services délégués.

Dans le cas de la dévolution, il faut assurer des ressources au niveau sn/l soit par l'attribution de champs fiscaux (Gilbert, 2009) ou soit par des transferts (Dafflon et Madiès, 2008). Bien qu'il soit préférable que des dépenses décidées localement soient financées localement du moins à la marge pour réduire le gaspillage et accroître l'imputabilité, ceci est, surtout pour les $s n / l$, rarement le cas en pratique. Il faut alors mettre sur pied un système de transferts ${ }^{1}$.

Un système de transferts intergouvernementaux doit répondre à deux questions : combien et comment transfère-t-on ? Il n'y a pas de réponse unique à ces questions, étant donné que chaque État a des objectifs différents de nature géographique (développement et intégration des régions frontalières pour assurer la sécurité nationale), politique (équilibre ethnolinguistique pour assurer l'harmonie et éviter ou réduire les tendances sécessionnistes) ou économique (assurer un niveau minimal ou égal de services sur l'ensemble du territoire, tenir compte des externalités possibles entre gouvernements locaux). Ceci dit :

- le montant total à transférer (combien) est déterminé de l'une des trois façons suivantes : i) un pourcentage fixe, soit de l'ensemble des recettes du gouvernement central, soit d'une partie des recettes tirées d'une ou de quelques taxes spécifiques; ii) un montant fixe déterminé annuellement comme toute autre dépense, avec ou sans consultation

\footnotetext{
${ }^{1}$ Cette section sur les transferts s'inspire de Bird et Vaillancourt (1998a)
} 
des gouvernements $s n / l$; iii) un montant variable déterminé par une formule (un pourcentage des dépenses des récipiendaires) ou un montant dépendant des caractéristiques de la population: (âge, éducation, etc.); ce dernier type de formule peut être envisagé pour financer la délégation. Chaque façon de faire a des avantages et des inconvénients. Ainsi, le pourcentage d'un impôt donné de l'État allant en transferts aux $s n / l$, s'il est élevé, réduit la rentabilité des efforts de perception du dit impôt par le gouvernement central et peut l'amener à les réduire. Par contre, les $s n / l$ peuvent, à assiette et efforts de perception inchangés, prévoir leurs recettes de transferts et donc emprunter sur cette base. Par ailleurs, la détermination annuelle du montant réduit la prévisibilité des recettes des sn/l mais permet, du moins en théorie, de mieux adapter les transferts à l'évolution des besoins. Ainsi, l'État, s'il se déleste de certaines dépenses, peut en assurer le financement à l'aide de transferts accrus.

- La répartition des transferts entre les récipiendaires. Les formules de transfert appartiennent à deux grands types. Certaines visent à garantir un niveau minimal similaire ou égal de services gouvernementaux dans toutes les régions d'un pays, en assurant aux gouvernements locaux des recettes adéquates. Ces formules, dites de péréquation, utilisent des indicateurs de la capacité fiscale et/ou des besoins des États. La mesure de ces deux types d'indicateurs est plus ou moins facile. Ainsi, il faut éviter de confondre assiette fiscale potentielle et assiette fiscale constatée - ou plus simplement, assiette et recettes. De même, il faut éviter de confondre niveau observé des dépenses et besoins.

D'autres formules visent à modifier le comportement des gouvernements locaux. Il s'agit alors de transferts conditionnels qui doivent être dépensés selon certains critères et dans certains domaines, accompagnés ou non de dépenses financées localement. Le premier type de transferts accroît la marge de manœuvre 
des gouvernements locaux, le second, non. L'à-propos de l'un ou l'autre type dépend à la fois de conditions objectives (capacité de gestion des gouvernements locaux, ...) et de la perspective retenue (« de haut en bas » ou « de bas en haut»). L'essentiel est de s'assurer que ces transferts n'ont pas d'effets pervers. Ainsi, un système de transferts assurant l'équilibre budgétaire, soit la couverture de la différence entre dépenses et recettes, peut décourager l'effort de perception fiscale par les gouvernements locaux. D'autres systèmes peuvent accroître certains types de dépenses, par exemple, des transferts couvrant le coût des salaires. Idéalement, les transferts auraient des effets incitatifs comme l'accroissement des efforts d'inscription au rôle fiscal ou de perception des impôts locaux.

Finalement, on doit noter que la disponibilité réelle des fonds plutôt que leur inscription d'une façon ou d'une autre dans un budget central, ou dans la caisse d'un agent comptable de l'état central agissant pour les sn/l au nom de l'unicité de caisse, est souvent l'obstacle le plus important à la décentralisation.

\section{Conclusion}

Une décentralisation significative nécessite un fort soutien de la part du gouvernement central. Ce soutien peut prendre diverses formes: octroi de la capacité normative, pouvoir autonome dans la délivrance des services à la population, collecte des recettes pour le financement.

La décentralisation place les autorités $s n / l$ en première ligne au niveau de la prestation des services. Cette nouvelle façon de voir les choses implique une identification des rôles de chaque niveau de l'état et des mécanismes à mettre en place pour permettre à chaque niveau de remplir leurs responsabilités respectives. Le principe de subsidiarité, c'est-à-dire, ce qui ne peut être fait à la base remonte au niveau immédiatement supérieur, suggère que cet examen soit conduit en commençant par le niveau le plus bas, le local, et remonte ainsi, jusqu'au niveau central.

En particulier, une décentralisation efficace requiert un gouvernement $s n / l$ fort. De plus, la capacité de gouvernance locale dépend largement de la capacité de 
collecter les recettes fiscales locales. À défaut, les instances sn/l resteront inévitablement dans un état de dépendance et de vulnérabilité, et la décentralisation n'aura pas de sens. Un niveau local fort requiert, quant à lui, la capacité d'édicter des normes. Les autorités régionales et locales doivent disposer de la capacité d'adopter librement les textes nécessaires.

Il est raisonnable de considérer qu'une période de transition bien structurée est nécessaire pour réaliser la décentralisation qu'elle que soit le niveau choisi. Les réformes centrales des systèmes de gestion du budget et du personnel doivent être approfondies et étendues à tous les niveaux, afin d'éviter les blocages et les rigidités, mais aussi pour permettre que les collectivités locales puissent atteindre l'autonomie qui leur est destinée. Seulement ceci améliorera la prestation des services de base et permettra une décentralisation efficace (Banque Mondiale, Bénin, 2002). Ceci dit, il faut faire attention de distinguer déconcentration d'appui et déconcentration de substitution. Ainsi, la déconcentration des services financiers de l'état qui permet un meilleur accès aux ressources financières par les $s n / l$ en est une d'appui. Par contre, déconcentrer des pouvoirs tels le développent économique alors que des $s n / l$ ont déjà des responsabilités dans ce secteur peut être une source de tension et de blocage. 


\section{Bibliographie}

Banque Mondiale. (2011). Decentralization \& Subnational Regional Economics. Consulté le 2 mars 2012, sur The World Bank.

Bird, R., \& Vaillancourt, F. (1998, septembre). Décentralisation financière et pays en développement : concepts, mesure et évaluation. L'Actualité économique, 74 (3), pp. 343 62.

Bird, R., \& Vaillancourt, F. (1998). Fiscal decentralization in Developing Countries : An Overview. Dans R. Bird, \& F. Vaillancourt, Fiscal Decentralization in Developing Countries (pp. 1-48). Cambridge : Cambridge University Press.

Bolderson, H., \& Mabbett, D. (1999). Devolved Social Security System: Principal Agent versus Multi-Level Governance. Journal of Public Politics (18), pp. 177-200.

Delcamp, A. (1995). Les problèmes de la déconcentration dans les pays européens. Revue française d'administration, 11 (4), pp. 730-40.

Dafflon, B. and Madiès, T. (2008), Décentralisation : quelques principes issus de la théorie du fédéralisme financier, Notes et documents no.42, Agence Française de Développement, Paris.

http://www.unifr.ch/finpub/assets/files/FedederalismefinancierN42.pdf

Falleti, T. G. (2005, août). A Sequential Theory of Decentralization: Latin American Cases in Comparative Perspective. The American Political Science Review, 99 (3), pp. 327-46.

Gerbaux, F., \& Pongy, M. (1994). Dysfonctionnements et incertitudes, décentralisation et politiques publiques locales. L'État de la décentralisation. Cahiers français, 256.

-Gilbert, G. (2009). Fiscalité locale : une grille de lecture économique. AFD Document de travail 87.

http://www.afd.fr/jahia/webdav/site/afd/shared/PUBLICATIONS/RECHERCHE/Scientif iques/Documents-de-travail/087-document-travail.pdf

Guengant, G. (2000). Développement des territoires et financement des collectivités locales.

Olowu, D. (1998). Évaluation des politiques de décentralisation en Afrique dans les années qutre-vingt-dix. Revue française d'administration publique (88), pp. 517-28.

Philippe, X. (1998). La répartition des compétences entre l'état central, l'état provincial et les municipalités : Structures politiques ou administratives. Revue française d'administration publique (85), pp. 15-33.

Rémond, B. (1999). La décentralisation aujourd'hui : un dessein politique sans dessin institutionnel. Les collectivités locales en mutation. Cahiers français (293), pp. 12-8.

Rosenbaum, A. (1998). Gouvernance et décentralisation : leçons de l'expérience. Revue française d'administration publique (88), pp. 507-16. 
Shah, A., \& Thompson, T. (2004). Implementing Decentralized Local Governance: A Treacherous Road with Potholes, Detours and Road Closures. World Banque Policy Research Working Paper 3353. World Bank.

Vaillancourt, François (2011) 'Decentralization in Madagascar: A String of Unfinished Races ' in Decentralization in Developing Countries Global Perspectives on the Obstacles to Fiscal Devolution ( Jorge Martinez -Vazquez and François Vaillancourt Ed) Northampton,Mass : Edward Elgar 2011 p359-388 